\title{
Criminologie
}

\section{Une comparaison de la délinquance des jeunes au Canada et aux États-Unis}

\author{
Jane B. Sprott et Howard N. Snyder
}

Volume 32, numéro 2, automne 1999

La justice des mineurs

URI : https://id.erudit.org/iderudit/004746ar

DOI : https://doi.org/10.7202/004746ar

Aller au sommaire du numéro

\section{Éditeur(s)}

Les Presses de l'Université de Montréal

ISSN

0316-0041 (imprimé)

1492-1367 (numérique)

Découvrir la revue

Citer cet article

Sprott, J. B. \& Snyder, H. N. (1999). Une comparaison de la délinquance des jeunes au Canada et aux États-Unis. Criminologie, 32(2), 55-82.

https://doi.org/10.7202/004746ar
Résumé de l'article

Cet article étudie les tendances de la délinquance juvénile au Canada et aux États-Unis pour la période entre 1991 et 1996. En ce qui concerne les infractions sérieuses commises avec violence, les infractions contre la propriété et les infractions liées à l'usage de la drogue, le Canada avait, en général, un plus faible taux d'arrestations que les États-Unis. Cependant, quand on observe les affaires qui ont reçu un traitement judiciaire formel, on se rend compte que le Canada avait des taux similaires ou à peine plus élevés que les États-Unis en ce qui a trait aux infractions avec violence et aux infractions contre la propriété. Aux États-Unis, la moitié des cas de délinquance juvénile échappait à la judiciarisation. Le Canada avait recours à la mise sous garde dans des proportions similaires ou un peu plus élevées que les États-Unis en ce qui concerne les infractions avec violence ou les infractions contre la propriété. C'est seulement pour les infractions reliées à la drogue que les États-Unis avaient un plus haut taux d'arrestations, d'affaires judiciarisées et de mises sous garde que le Canada. Les États-Unis transféraient aussi les cas de mineurs aux tribunaux pour adultes dans des proportions beaucoup plus élevées. Enfin, il n'est pas établi que les infractions avec violence commises par les jeunes aient augmenté tant au Canada qu'aux États-Unis entre 1991 et 1996. La plus grande " augmentation de crimes » concernait les infractions reliées à la drogue.
Tous droits réservés @ Les Presses de l'Université de Montréal, 1999
Ce document est protégé par la loi sur le droit d'auteur. L'utilisation des services d'Érudit (y compris la reproduction) est assujettie à sa politique d'utilisation que vous pouvez consulter en ligne.

https://apropos.erudit.org/fr/usagers/politique-dutilisation/ 


\title{
Une comparaison de la délinquance des jeunes au Canada et aux États-Unis ${ }^{1}$
}

\author{
Jane B. Sprott \\ Chercheure \\ National Center on Addiction and Substance Abuse \\ Columbia University \\ New York \\ j.sprott@utoronto.ca
}

Howard N. Snyder

Directeur

Systems Research

National Center for Juvenile Justice

Pittsburgh

snyder@ncjj.org

RÉSumÉ - Cet article étudie les tendances de la délinquance juvénile au Canada et aux États-Unis pour la période entre 1991 et 1996. En ce qui concerne les infractions sérieuses commises avec violence, les infractions contre la propriété et les infractions liées à l'usage de la drogue, le Canada avait, en général, un plus faible taux d'arrestations que les États-Unis. Cependant, quand on observe les affaires qui ont reçu un traitement judiciaire formel, on se rend compte que le Canada avait des taux similaires ou à peine plus élevés que les États-Unis en ce qui a trait aux infractions avec violence et aux infractions contre la propriété. Aux États-Unis, la moitié des cas de délinquance juvénile échappait à la judiciarisation. Le Canada avait recours à la mise sous garde dans des proportions similaires ou un peu plus élevées que les États-Unis en ce qui concerne les infractions avec violence ou les infractions contre la propriété. C'est seulement pour les infractions reliées à la drogue que les États-Unis avaient un plus haut taux d'arrestations, d'affaires judiciarisées et de mises sous garde que le Canada. Les États-Unis transféraient aussi les cas de mineurs aux tribunaux pour adultes dans des

1. La préparation de cet article a été partiellement rendue possible par une subvention de recherche du Conseil de recherches en sciences humaines du Canada au professeur A. N. Doob. 
proportions beaucoup plus élevées. Enfin, il n'est pas établi que les infractions avec violence commises par les jeunes aient augmenté tant au Canada qu'aux États-Unis entre 1991 et 1996. La plus grande « augmentation de crimes » concernait les infractions reliées à la drogue.

ABSTRACT - This paper investigates trends in youth crime from 1991 to 1996 in Canada and the United States. Generally, Canada had lower arrest rates than the United States for serious violent offences, property and drug offences. However, when looking at the formal juvenile court processing of cases, Canada had similar, or slightly higher rates than the US for all violence and all property cases. The United States diverted about half of the cases from the formal juvenile court process. Canada used custody at a similar, or slightly higher rate than the US for violent offences and property offences. Only in drug related offences did the United States have higher arrest and court processing rates, and used custody at a higher rate than Canada. The United States transferred cases to adult court at a substantially higher rate than Canada. Overall, there appeared to be no evidence that serious violent youth crime had increased in either Canada or the United States between 1991 and 1996. The greatest "crime increase" for both countries was in drug related offences.

\section{Introduction}

Au Canada et aux États-Unis, les sondages d'opinion révèlent que, d'après la plupart des gens, les crimes et spécialement les crimes commis par les jeunes sont en augmentation. Par exemple, en 1990, 47 \% des Canadiens interrogés pensaient que le comportement des jeunes avait empiré en quelques années. En 1993, 64 \% des Canadiens interrogés partageaient la même opinion (Robert et Stalans, 1997). Lors d'une enquête récente menée en Ontario, 79 \% des répondants croyaient que le nombre de jeunes accusés d'homicide avait augmenté au fil des années (Doob et al., 1998). On retrouve les mêmes perceptions aux États-Unis (Robert et Stalans, 1997). En outre, le public estime non seulement que les sentences infligées aux jeunes sont trop clémentes (Doob et al., 1998), mais aussi que les tribunaux pour mineurs sont devenus plus indulgents ces dernières années (Robert et Stalans, 1997).

En mai 1998, la ministre canadienne de la Justice annonçait qu'elle allait élaborer une nouvelle législation concernant la justice des mineurs. Se fondant sur les impressions du public à l'égard d'une justice estimée 
trop clémente, la ministre affirmait que les gens avaient «perdu confiance » dans la législation en vigueur sur les jeunes contrevenants. Elle affirmait aussi que « la proportion d'adolescents incarcérés au Canada est beaucoup plus élevée que dans bon nombre de pays occidentaux, dont les États-Unis... » (McLellan, 1998 : 8). Malheureusement, elle ne fournissait aucune donnée chiffrée pour soutenir une telle affirmation.

De plus, affirmer simplement que le Canada a « un taux plus élevé » d'incarcération des mineurs que les États-Unis masque la complexité inhérente à toute étude comparée entre pays.

Dans le présent article, nous poursuivons un double objectif. En premier lieu, nous désirons mettre en lumière les complexités méthodologiques que comporte la comparaison entre deux pays. Deux problèmes méthodologiques se posent alors immédiatement. Tout d'abord, les comparaisons nationales empêchent de tenir compte des variations entre provinces ou entre états à l'intérieur de chaque pays. Ensuite, le Canada et les États-Unis ne placent pas les même catégories d'âge sous la compétence de la justice des mineurs.

Tout en tenant compte des complexités méthodologiques, notre second objectif est d'étudier le volume, la nature et les tendances qui caractérisent la délinquance juvénile ainsi que les réactions que la justice criminelle du Canada et des États-Unis lui oppose. En procédant de la sorte, nous ne désirons pas seulement explorer les différences et les ressemblances existant entre le Canada et les États-Unis, nous entendons aussi vérifier si la délinquance juvénile officiellement enregistrée est en augmentation et si la justice criminelle envers la délinquance des jeunes est devenue « plus indulgente » au fil des ans.

Afin de saisir les tendances caractérisant la délinquance juvénile officiellement répertoriée dans les deux pays, nous avons étudié les taux d'arrestations concernant les infractions avec violence, les infractions contre la propriété et les infractions reliées à la drogue pour la période 1991-1996. Nous avons aussi analysé la réaction légale à la délinquance juvénile pour la période 1991-1995 en recherchant dans quelles proportions les infractions avec violence, les infractions contre la propriété et les infractions liées à la drogue avaient été amenées au tribunal de la jeunesse, et dans quelle mesure on avait eu recours aux mesures de rechange, à la mise sous garde et aux renvois aux tribunaux pour adultes. Nous avons débuté notre étude à partir de 1991 parce que c'est à compter de cette année que toutes les provinces canadiennes participè- 
rent à la collecte de données relatives au tribunal de la jeunesse. On ne pouvait donc évaluer de façon acceptable l'activité des tribunaux de la jeunesse au Canada avant ce moment. Nous avons arrêté notre analyse en 1995-96 parce que les données les plus récentes dont nous disposions pour les États-Unis au moment de rédiger cet article concernaient cette période.

\section{Difficultés méthodologiques}

\section{Variations entre provinces et entre États}

Au Canada, le fait d'appliquer une loi fédérale unique (la Loi sur les jeunes contrevenants) n'empêche pas l'existence d'importantes variations entre provinces. Par exemple, on constate des différences entre provinces dans le taux de judiciarisation et dans le taux de mise sous garde (Doob et Sprott, 1996 et 1999).

Le Tableau 1 présente les variations existant entre quatre provinces sélectionnées (qui totalisent $83 \%$ de la population des jeunes du groupe d'âge visé par la loi) en ce qui concerne les taux de mises en accusation et de mises sous garde. Alors qu'au Canada le taux (pour 1000 jeunes âgés de 12 à 17 ans) de judiciarisation au tribunal de la jeunesse est de 45,3 , on observe une variation considérable entre les provinces sélectionnées, allant du taux le plus bas au Québec $(18,8)$ au plus élevé en Alberta $(65,3)$. On constate le même type de variation en ce qui concerne l'imposition de mises sous garde. L'Ontario impose la mise sous garde dans $40,7 \%$ des cas recevant une sentence, tandis que l'Alberta y a recours dans $27,9 \%$ des cas. En observant les taux de mises sous garde pour 1000 jeunes dans la population (de chaque province), on constate aussi une variation. Alors que le taux de mises sous garde pour l'ensemble du Canada est de 10,5 pour 1000 jeunes, celui du Québec est beaucoup plus bas $(4,4)$ et le taux en Alberta est un peu plus élevé $(12,7)$.

Aux États-Unis, il existe aussi de fortes variations parce que le système de justice des mineurs est élaboré en grande partie par chaque État. Par exemple, alors que la plupart des États considèrent les jeunes comme des adultes (dans le cadre de la justice criminelle) à l'âge de 18 ans, certains États fixent l'âge adulte à 16 ou 17 ans. De plus, dans beaucoup d'États, les jeunes qui commettent certains crimes violents graves (qui varient suivant les États) sont automatiquement considérés comme des adultes et placés sous la juridiction de tribunaux pour adultes. En ce 
T A B LE A U 1

Recours au tribunal et à la mise sous garde :

Canada et les provinces les plus importantes, 1997-1998.

\begin{tabular}{|lccc|}
\hline & $\begin{array}{c}\text { Cas portés au tribunal } \\
\text { pour 1 000 jeunes } \\
\text { (âgés de 12 à 17 ans) } \\
\text { dans la population }\end{array}$ & $\begin{array}{c}\text { Pourcentage de } \\
\text { personnes trouvées } \\
\text { coupables et mises } \\
\text { sous garde }\end{array}$ & $\begin{array}{c}\text { Cas mis sous garde } \\
\text { pour 1 000 jeunes } \\
\text { (âgés de 12 à 17 ans) } \\
\text { dans la population }\end{array}$ \\
\hline CANADA & 45,3 & 34,4 & 10,5 \\
QUÉBEC & 18,8 & 28,9 & 4,4 \\
ONTARIO & 49,3 & 40,7 & 12,3 \\
ALBERTA & 65,3 & 27,9 & 12,7 \\
COLOMBIE- & 42,3 & 33,1 & 9,7 \\
BRITANNIQUE & & & \\
\hline
\end{tabular}

sens, il n'y a donc pas un seul, mais 51 systèmes de justice des mineurs aux États-Unis (pour de plus amples détails sur la variation entre États, voir Snyder et Sickmund, 1995). Ainsi, en se concentrant sur les seules données nationales, on ne peut percevoir l'existence des variations entre provinces et entre États. De même, une telle perspective empêche de réaliser à quel point les systèmes de justice des mineurs du Canada et des États-Unis sont fort différents.

\section{Différentes catégories d'âge}

Aux États-Unis, les enfants peuvent être officiellement inculpés et pris en charge par le système de justice des mineurs à compter de l'âge de sept ans, alors qu'au Canada la catégorie d'âge visée par ces mesures est celle des 1217 ans. Il ne nous était pas possible de distinguer les arrestations d'enfants de moins de douze ans de l'ensemble des données relatives aux arrestations des jeunes aux États-Unis : nous devions donc aborder cette question sous un autre angle. Nous avons essayé de rendre les données du Canada comparables à celles des États-Unis en estimant quel serait le nombre d'enfants âgés de 10 et 11 ans qui pourraient être arrêtés au Canada. Cette démarche s'avéra cependant difficile ${ }^{2}$. Donc, nous avons plutôt rendu les données relatives aux États-Unis comparables à celles du Canada.

2. Nous avons essayé de trouver un modèle statistique pouvant convenir aux distributions de fréquences des accusations de délits violents et de délits contre la propriété pour les jeunes âgés de 12 à 17 ans, de façon à pouvoir ensuite estimer statistiquement quel serait le nombre d'enfants de 10 à 11 ans susceptibles d'être accusés de ces types de délit. Lorsqu'on utilise les fréquences, l'hypothèse la plus simple est qu'une distribution de Poisson 
Pour calculer les taux d'arrestations au Canada, nous avons divisé le nombre de jeunes âgés de 12 à 17 ans qui furent arrêtés par le nombre de jeunes de 12 à 17 ans dans la population. Pour les États-Unis, nous avons utilisé comme numérateur le nombre de jeunes arrêtés âgés de 17 ans et moins, et nous avons divisé ce chiffre par le nombre de jeunes âgés de 12 à 17 ans dans la population. Les taux d'arrestations obtenus pour les États-Unis sont en conséquence probablement plus élevés de $3 \%$ que si nous avions utilisé comme numérateur le nombre d'arrestations de jeunes âgés de 12 à 17 ans. Cela devrait être pris en compte lors de l'interprétation des données relatives aux arrestations.

Lors de l'étude de l'activité des tribunaux, nous avons dû recourir à un dénominateur différent. Pour les États-Unis, nous avons employé comme dénominateur le nombre de jeunes dont l'âge va de 10 ans jusqu'à l'âge maximal pouvant relever du tribunal pour mineurs. Ce type de dénominateur visait à corriger le problème lié à la variation des âges maximums admissibles suivant les États. Le numérateur regroupait l'ensemble des jeunes de 17 ans et moins qui avaient été pris en charge par le système de justice des mineurs. Pour calculer un taux comparable au Canada, nous avons divisé le nombre de jeunes âgés de 17 ans et moins qui étaient passés au tribunal de la jeunesse par le nombre de jeunes âgés de 10 à 17 ans $^{3}$.

pourra caractériser cette distribution. Pour les accusations d'infraction avec violence en 1996-1997, une distribution de Poisson a effectivement correspondu à la courbe des crimes et ainsi a pu prédire, de façon relativement précise, le nombre d'accusations pour chaque catégorie d'âge. De ce fait, nous avons aussi pu utiliser ce modèle pour prédire les accusations susceptibles de toucher la catégorie des jeunes de 10-11 ans. Pour les infractions avec violence des années 1991-1992 à 1995-1996, la correspondance était adéquate. Cependant, en ce qui concerne les distributions relatives aux infractions contre la propriété au cours des années, nous n'avons pas trouvé de modèle réaliste susceptible de convenir à la distribution. Le problème, en l'occurrence, est qu'on ne pouvait faire que des extrapolations sur la base de données dont nous ne disposions pas. De ce fait, diverses hypothèses proposées pouvaient entraîner des résultats différents, tous essentiellement invérifiables. Et même si une telle analyse s'était avérée possible, il aurait fallu accomplir un travail considérable pour produire des modèles correspondant aux distributions de fréquences des infractions avec violence et des infractions contre la propriété en fonction de toutes les catégories d'âge et pour toutes les années. Nous tenons à remercier le professeur D. A. Sprott du Centro de Investigaciones en Matemáticas de Guanajuato, au Mexique, pour ses conseils en matière de statistiques.

3. En considérant la population de jeunes âgés de 10 à 17 ans comme dénominateur pour calculer le taux d'activité du tribunal de la jeunesse au Canada, on peut procéder à des comparaisons intéressantes avec les États-Unis. Cependant les chiffres ainsi obtenus ne devraient pas être considérés comme les taux réels d'activité du tribunal de la jeunesse au Canada. Pour obtenir de tels taux, il faudrait que le dénominateur représente la population de jeunes admissibles au tribunal de la jeunesse (âgés de 12 à 17 ans). 
Ces problèmes que soulèvent les variations interprovinciales et interétatiques ainsi que les différences de seuils d'âge entre les deux pays sont-ils suffisamment importants pour empêcher toute comparaison entre les deux pays? Il y a là une question qui, comme pour la notion de beauté, dépend du regard de chacun. À notre avis, il est possible d'obtenir une compréhension valable et utile des différentes similarités des deux pays à la condition de garder à l'esprit l'existence de certaines limites d'ordre méthodologique.

\section{Le volume, la nature et l'évolution de la délinquance juvénile au Canada et aux États-Unis}

Pour cette section, nous avons étudié les données relatives aux arrestations effectuées par la police. Au Canada, ces données proviennent en grande partie des statistiques canadiennes de la criminalité, publiées annuellement par Statistique Canada. Les données d'arrestations par la police représentent le nombre de jeunes que la police inculpe ou pour lesquels elle recommande l'inculpation. Dans certains cas, on peut par la suite changer le chef d'inculpation ou l'abandonner, tout comme certaines affaires peuvent aussi avoir été déclarées non fondées ou avoir été diverties du système judiciaire. On constatera sur tous les tableaux une assez forte chute des taux d'arrestations pour le Canada en ce qui concerne tous les crimes entre 1991 et 1992. Ceci est en partie dû au fait que Toronto ne commença à enregistrer les délits en fonction du chef d'accusations le plus grave qu'à partir de 1992. Auparavant, Toronto employait une méthode de calcul tenant compte de multiples délits. Toronto représente environ $10 \%$ de la population du pays. Les données concernant le taux d'arrestations des jeunes aux États-Unis proviennent de données recueillies et rapportées à l'origine par le Federal Bureau of Investigation (FBI). Des estimations nationales des arrestations de jeunes et les taux d'arrestations des jeunes sont tirées de statistiques provenant d'échantillons du FBI et sont publiées par le ministère de la Justice des États-Unis (Snyder, 1997).

Nous avons d'abord étudié le nombre de jeunes (de 12 à 17 ans) portés à l'attention de la police (pour tous les délits sauf les infractions à la circulation routière $)^{4}$. On constate que le taux obtenu pour le Canada

4. Pour le Canada, ces données incluent aussi les « jeunes non-inculpés ». Cependant, on ignore exactement quelles personnes la police inclut dans la catégorie « jeunes non 
est un peu plus bas que celui des États-Unis (Figure 1). Au Canada, le taux d'arrestations de jeunes a diminué jusqu'en 1993 et est resté relativement stable depuis lors. Par contre, aux États-Unis, on a constaté une légère augmentation du nombre de jeunes dont les cas ont été portés à la connaissance de la police. Il résulte de ces tendances opposées que, en 1991, le taux de cas portés à la connaissance de la police au Canada était environ $8 \%$ plus bas que celui des États-Unis alors qu'en 1996, il était plus bas de $30 \%$.

La violence. - En ce qui concerne le taux d'arrestations pour homicide (Figure 2), on constate que le Canada a un taux plus bas que les États-Unis. En 1996, le taux d'arrestations pour homicide au Canada était de 2,07 pour 100000 jeunes âgés de 12 à 17 ans tandis que le taux aux États-Unis était de 13,39. Bien qu'au Canada ce taux soit resté assez stable au cours des années, les taux concernant les États-Unis ont atteint un sommet en 1993 et ont diminué depuis lors.

Les États-Unis disposent d'un «index des crimes violents »: le meurtre, l'agression sexuelle, le vol qualifié et les voies de fait graves. Afin d'établir un index comparable pour le Canada, il fallut intégrer le meurtre, l'agression sexuelle grave, l'agression sexuelle sous la menace d'une arme, l'agression sexuelle, le vol qualifié, les voies de fait graves et les voies de fait avec utilisation d'une arme. En regardant la Figure 3, nous voyons que le taux d'arrestations pour ce type d'infractions avec violence est constamment plus faible au Canada qu'aux États-Unis. Ainsi, en 1996, le taux d'arrestations au Canada pour ces infractions avec violence était de 38 \% plus bas que celui des États-Unis. Donc, en ce qui concerne ces infractions avec violence, le Canada semble avoir eu un taux d'arrestations assez stable, tandis que les États-Unis ont vu leur taux augmenter jusqu'aux environs de 1994 et diminuer légèrement depuis lors.

inculpés ». Pour une juridiction donnée, cette catégorie peut regrouper ou non des jeunes référés à des mesures de rechange, et/ou certains jeunes âgés de moins de 12 ans. De plus, certaines juridictions (comme celle de Toronto) n'enregistreraient pas le nombre de « jeunes non inculpés». On ignore dans quelles proportions les autres forces de police n'enregistrent pas le nombre de jeunes «non inculpés ». Il faudrait tenir compte de cela lors de l'interprétation des données. Ces données ont été obtenues grâce à une demande spéciale à Statistique Canada. En ce qui concerne les taux d'arrestations pour les autres infractions que nous avons étudiées (violence grave, infractions contre la propriété et infractions reliées à la drogue), nous n'avons pas inclus le nombre de jeunes «non inculpés ». Cela était lié au fait que nous nous intéressions aux jeunes qui avaient été inculpés, ou pour lesquels la police avait recommandé l'inculpation, ce qui rendait possible leur acheminement dans le système de justice des mineurs. 


\section{F I G U RE 1}

Taux de jeunes dont le cas a été porté à la connaissance de la police (pour toutes les infractions)

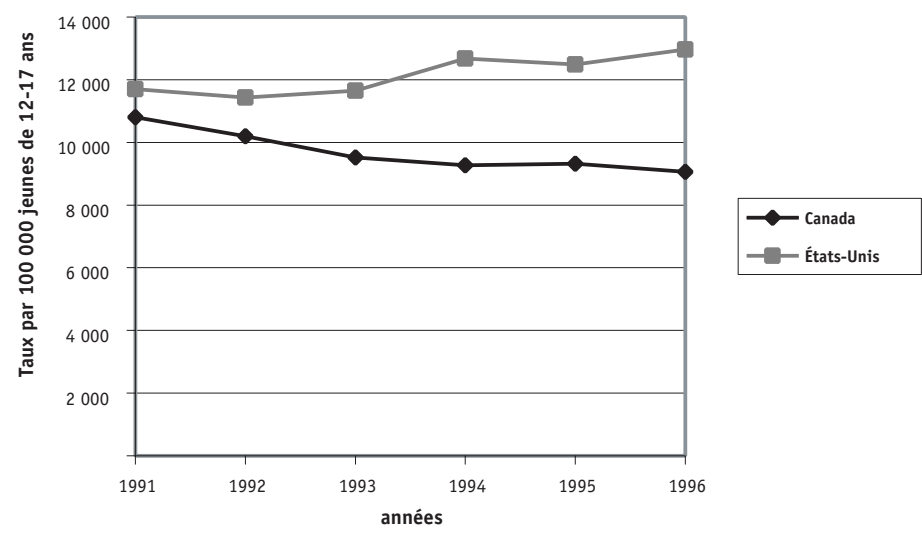

FIG URE 2

Taux d'arrestations pour homicide

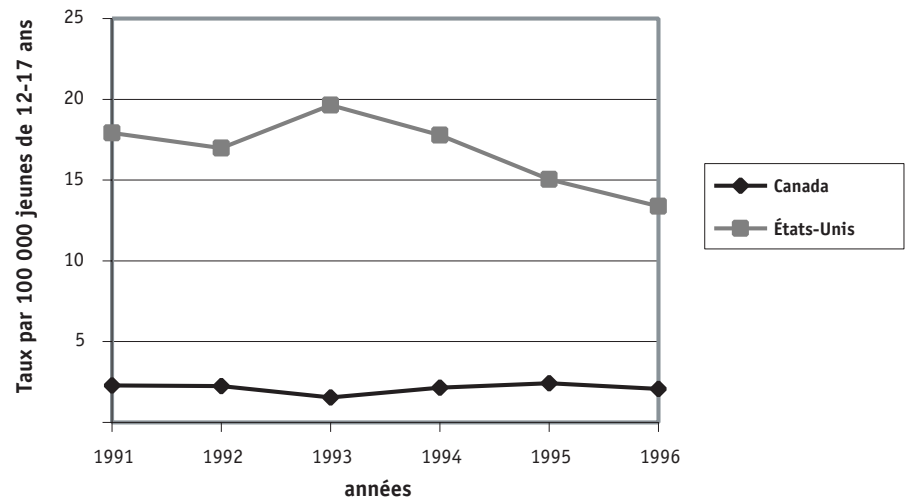


F I G URE 3

Taux d'arrestations pour infractions avec violence relativement sérieuses

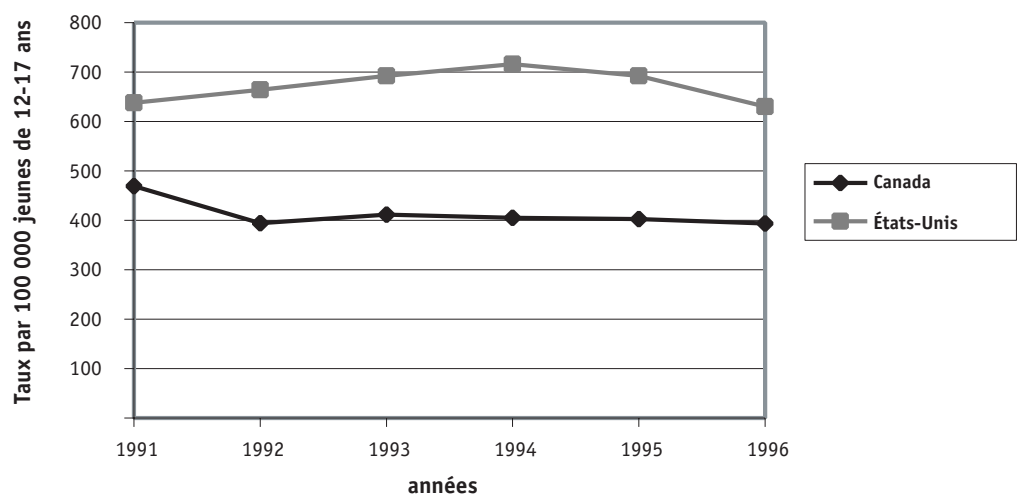

Infractions contre la propriété. - Nous avons ensuite étudié les taux d'infractions contre la propriété au Canada et aux États-Unis. Les ÉtatsUnis disposent aussi d'un « index des crimes contre la propriété » dans lequel sont répertoriés le cambriolage, le vol, le vol de véhicules motorisés et l'incendie criminel. Pour établir un index à peu près semblable concernant le Canada, nous avons intégré l'introduction par effraction, le vol (d'une valeur de plus ou de moins de 5000 \$), le vol d'un véhicule motorisé et l'incendie criminel. D'après la Figure 4, nous voyons que les taux d'arrestations pour ces infractions sont restés assez stables aux États-Unis durant les cinq dernières années. Il y a une très forte baisse des arrestations au Canada entre 1991 et 1992 ; cependant, on ignore si cette forte baisse reflète la réalité ou si elle n'est pas plutôt le résultat de changements administratifs, tel que l'abandon de la prise en compte d'infractions multiples (multiple offence scoring) que nous avons men-tionné précédemment. En général, les taux d'arrestations canadiens pour ce type d'infractions semblent cependant être plus bas d'environ $27 \%$ par rapport aux États-Unis.

En observant les taux d'arrestations pour diverses infractions au fil des années, il ne semble pas y avoir d'indication que la délinquance juvénile ait augmenté dramatiquement entre 1991 et 1996, que ce soit au Canada ou aux États-Unis. Même si le Canada a eu des taux d'arrestations plus bas que les États-Unis, on n'a pas non plus remarqué de hausse substantielle de ces infractions aux États-Unis. En effet, après avoir atteint des 


\section{FIG URE 4}

Taux d'arrestations pour certaines infractions contre la propriété

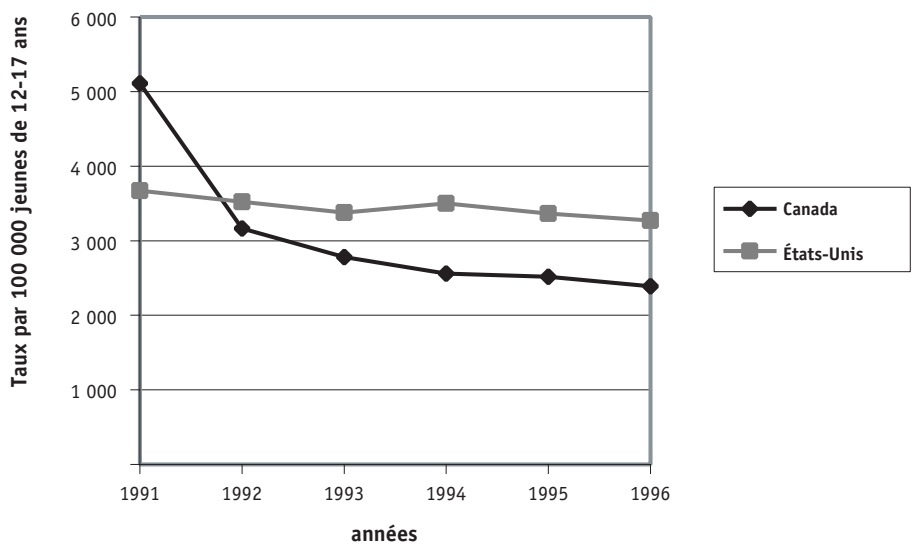

\section{F I G URE 5}

Taux d'arrestations pour les infractions reliées à la drogue

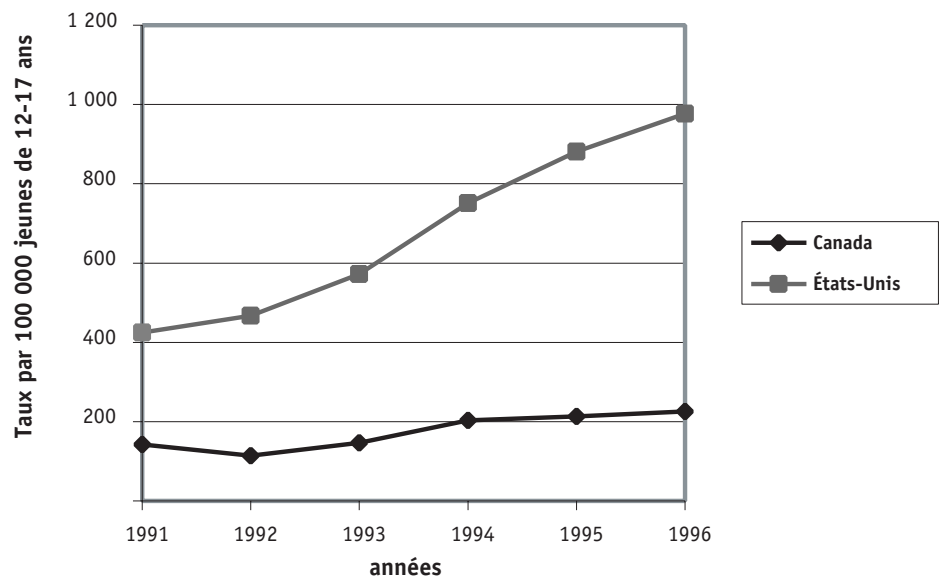


sommets aux environs de 1993-1994, les taux d'arrestations ont baissé aux États-Unis. Néanmoins, il faut remarquer que les taux d'arrestations pour crimes violents aux États-Unis durant les années 1990 sont substantiellement plus élevés que ceux des années 1980. Toujours aux ÉtatsUnis, la période 1987-1994 a connu des augmentations significatives du taux d'arrestations de mineurs pour les infractions avec violence.

Les drogues. - Dans le domaine des délits reliés à la drogue, on pourrait s'attendre à observer une augmentation des arrestations, au moins pour les États-Unis. Durant la dernière décennie, les États-Unis ont lancé une " guerre contre la drogue », entraînant de nouvelles stratégies en matière d'application de la loi et de nouvelles politiques de sentencing (Tonry, 1997). En conséquence, comme nous pouvons l'observer à la Figure 5, il y a eu une très forte augmentation du taux d'arrestations pour délits de drogue à partir de 1992 aux États-Unis. Au Canada, bien qu'il y ait eu une certaine augmentation dans les taux d'arrestations pour délits de drogue, le phénomène a eu nettement moins d'ampleur qu'aux États-Unis.

\section{La « réaction légale " à la délinquance juvénile au Canada et aux États-Unis}

Dans cette section, nous allons étudier la manière selon laquelle les cas sont traités dans le système de justice des mineurs. Pour le Canada, nous tenons compte habituellement du chef principal d'accusation, le motif le plus grave pour lequel le cas est amené au tribunal. Ces données ont été fournies par le Centre canadien de la statistique juridique qui produit annuellement les statistiques des tribunaux pour le Canada et les provinces. Pour les États-Unis, nous avons utilisé l'équivalent du principal chef d'accusation. Comme les États-Unis ne récoltent pas de données de toutes les agences de justice criminelle, nous nous sommes fondés sur des estimations nationales (Snyder et al.,1997) ${ }^{5}$.

5. Les statistiques des tribunaux de la jeunesse ont été obtenues à partir des données des Archives nationales des tribunaux (National Court Data Archive). Ce bureau d'archives recueille des données qui lui sont fournies par les agences des États et des comtés qui sont responsables de la collecte et de la distribution d'informations relatives au cheminement des mineurs dans les tribunaux de la jeunesse. Ces données ne sont pas le produit d'un programme uniforme de collecte de données, ni d'un recensement complet effectué auprès des tribunaux de la jeunesse. Ces estimations ont été mises au point en utilisant des informations compatibles provenant de tous les tribunaux qui étaient en mesure de fournir des données au bureau d'archives. Pour une discussion plus complète sur ces données et leurs limites, voir la section méthodologique de Juvenile Court Statistics 1995 (Sickmund et al., 1998). 
Quand un cas est renvoyé au système judiciaire aux États-Unis, il peut être traité suivant une procédure «formelle » ou une procédure «informelle ». Suivre la filière «formelle» signifie que le cas est porté devant le juge qui doit tout d'abord statuer sur la culpabilité. Aux ÉtatsUnis, le tribunal ne considère pas un jeune coupable d'une infraction particulière ; le jeune est plutôt déclaré « délinquant ». Si un jeune est reconnu délinquant dans le cadre de la filière formelle, le tribunal impose des sanctions au jeune. Parmi les sanctions disponibles, on trouve le placement en résidence (mise sous garde), la probation ou la restitution à la victime. À l'opposé, dans certains cas, des personnes responsables de l'admission au tribunal (des procureurs ou du personnel de la probation) peuvent décider que le cas soit traité sans avoir recours à une audition formelle. Ces cas sont traités suivant la voie informelle et, si l'affaire n'est pas classée, le jeune accepte «volontairement» une mesure proposée par la cour. Si le jeune ne se plie pas aux sanctions informelles, le cas est alors amené devant un juge suivant une procédure formelle. Les mesures « informelles » ressemblent donc aux mesures de rechange du Canada.

\section{Les cas présentés au tribunal de la jeunesse}

Nous avons étudié les cas référés par la police et qui ont comparu devant un tribunal. Le lecteur se rappellera cependant qu'un tel type de comparaison nationale ne tient pas compte des variations entre provinces et entre États. Pour les États-Unis, nous n'avons analysé que les cas ayant suivi la filière « formelle». Nous n'avons pas tenu compte des cas traités de façon informelle parce que même s'ils étaient référés au système de justice des mineurs, ils étaient écartés du processus judiciaire.

Comme nous l'avons mentionné précédemment, nous avons calculé le taux pour le Canada en divisant le nombre de jeunes âgés de 17 ans et moins inculpés formellement par le nombre total de jeunes âgés de 10 à 17 ans au sein de la population. En ce qui concerne les États-Unis, nous avons calculé le taux en divisant le nombre de jeunes de 17 ans et moins traités «formellement» par le nombre total de jeunes ayant 10 ans et plus dans la population, l'âge maximum dans ce cas étant celui qui est à la limite de l'admissibilité au tribunal de la jeunesse (l'âge de la majorité pénale). Pour plus de simplicité, les taux sur les figures sont présentés comme des taux pour 100000 jeunes âgés de 10 à 17 ans. Nous avons tenu compte des infractions avec violence, des infractions contre la propriété et des infractions liées à la drogue. Cette fois-ci 
cependant, au lieu de nous fonder sur l'index des crimes violents et l'index des crimes contre la propriété, nous avons pris en compte tous les cas de violence et tous les cas d'atteinte à la propriété.

En abordant l'analyse des crimes violents, nous avons remarqué que le Canada avait, jusqu'à récemment, porté de tels cas devant les tribunaux à un taux plus élevé que les États-Unis (Figure 6). Les cas de violence ont augmenté aux États-Unis au fil des années tandis qu'ils sont restés relativement stables au Canada. Cependant, comme notre étude aborde toute sorte de violence, une augmentation peut être due à un plus grand nombre d'actes violents de moindre importance comme des voies de fait mineures. D'après estimation, la majorité des cas de violence répertoriés aux États-Unis consiste en des voies de fait simples (Stahl, 1998). La situation est la même au Canada, où il semble que toute augmentation de la violence soit due à un accroissement des agressions mineures plutôt qu'à un accroissement de formes plus graves de violence (Doob et Sprott, 1998).

Si l'on considère maintenant l'ensemble des infractions contre la propriété, on constate que le Canada renvoie ce type de cas au tribunal de la jeunesse dans de plus fortes proportions que les États-Unis (Figure 7). Cependant, alors que les taux sont demeurés relativement stables aux États-Unis, ils ont diminué au Canada. Ainsi, en 1995, les deux pays portaient ces délits devant les tribunaux à des taux à peu près identiques.

En ce qui concerne les délits de drogue, nous observons que les ÉtatsUnis ont un plus haut taux de mises en accusation que le Canada (Figure 8). Les deux pays ont connu une augmentation de la judiciarisation de tels cas. Néanmoins, il faut noter qu'au fil des années, le Canada a présenté au tribunal des cas de délits de drogue à un taux de moitié plus faible que celui des États-Unis.

Si le Canada a porté au tribunal un peu plus de cas (de violence et d'atteinte à la propriété) que les États-Unis, il ne faut pas oublier que les États-Unis traitent ces cas suivant une procédure soit formelle, soit informelle. Or nous avons seulement analysé les cas qui ont suivi la filière formelle. En considérant tous les cas qui sont passés par les tribunaux aux États-Unis, nous avons constaté qu'environ la moitié d'entre eux furent traités de manière informelle (Figure 9). Au fil des années, on constate une augmentation du recours aux procédures formelles. Cette tendance se vérifie en matière de violence, d'atteinte à la propriété et de drogue. Le Canada a aussi recours à des «mesures de rechange » en vue d'éviter la judiciarisation, mais il est peu probable que son taux de diversion approche celui des États-Unis. 


\section{FIG URE 6}

Taux de judiciarisation au tribunal de la jeunesse pour toutes les infractions avec violence
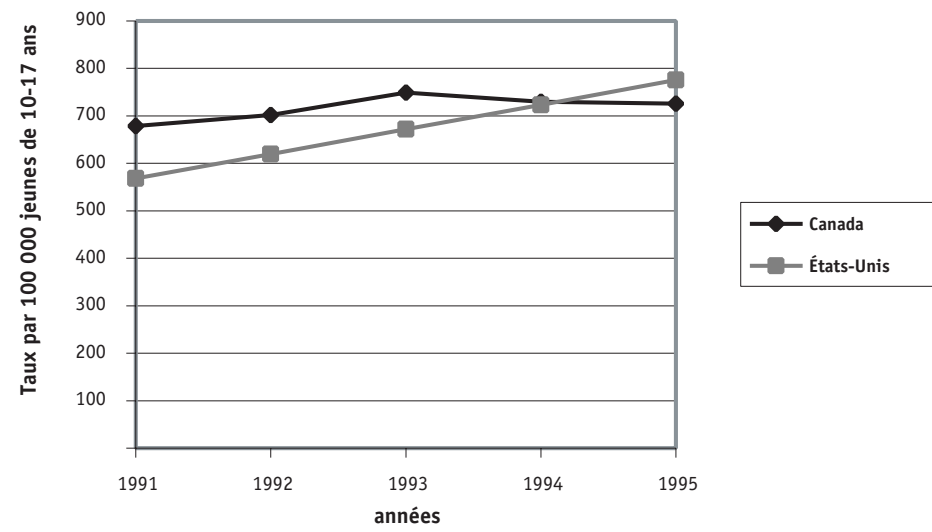

F I G URE 7

Taux de judiciarisation au tribunal de la jeunesse pour toutes les infractions contre la propriété

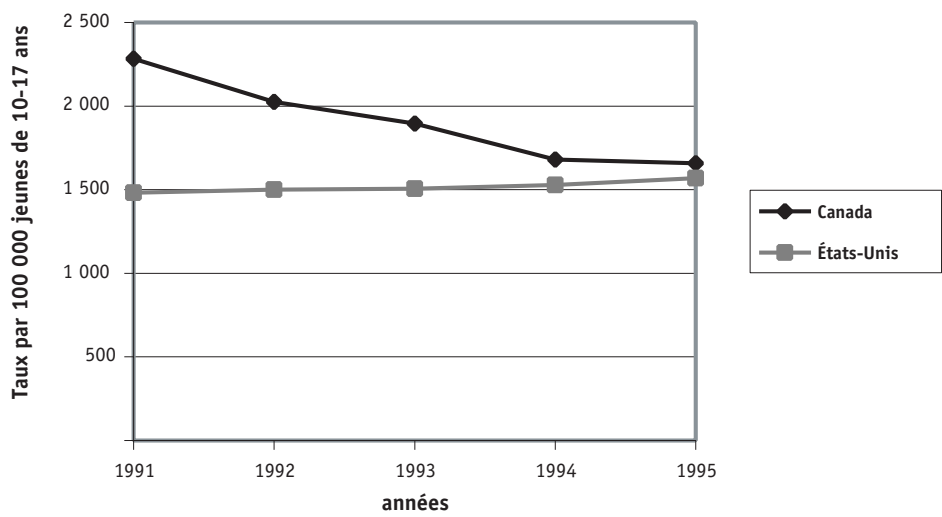




\section{FIGURE 8}

Taux de judiciarisation au tribunal de la jeunesse pour toutes les infractions reliées à la drogue

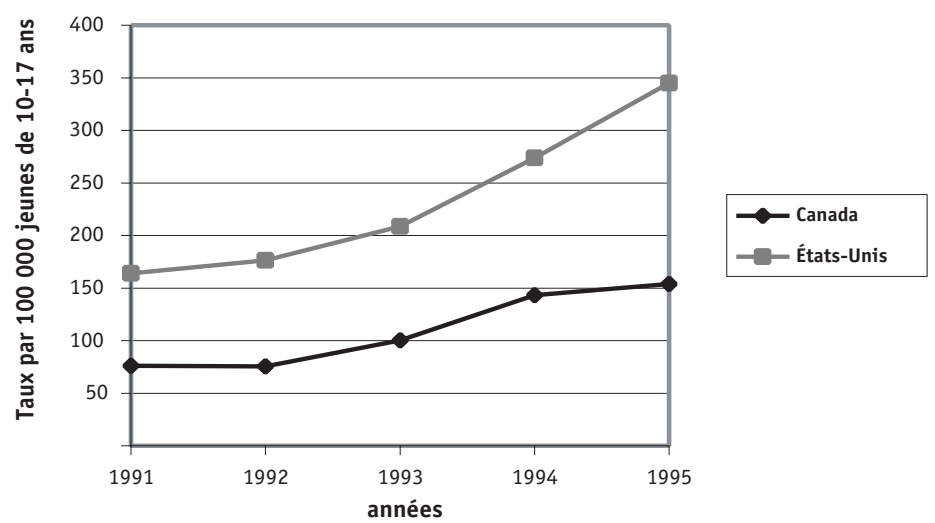

La seule mesure de non-judiciarisation disponible au Canada provient des rapports de police, dans la catégorie des jeunes "non inculpés ». Comme mentionné dans la note $\mathrm{n}^{\circ} 3$, la catégorie des « jeunes non inculpés » peut ou non regrouper les jeunes référés aux programmes de mesures de rechange et/ou certains jeunes âgés de moins de 12 ans. De plus, certaines juridictions comme Toronto n'enregistrent pas le nombre de jeunes qui n'ont pas été inculpés. On ne dispose pas non plus d'estimations nationales quant au nombre de jeunes dirigés vers les programmes de mesures de rechange, par conséquent, nous n'avons pu les inclure dans nos calculs. Dans un tel contexte, notre mesure de non-judiciarisation (des « jeunes non inculpés ») sousévalue le nombre de jeunes ayant évité la judiciarisation au Canada. Cependant, en observant les taux d'arrestations et de « non-inculpation » par la police, nous constatons que le taux d'arrestation a un peu diminué au fil des années tandis que le taux de «non-inculpation » est resté relativement stable (Figure 10) ${ }^{6}$.

Déclaration de culpabilité. - Par rapport aux États-Unis, le Canada envoie au tribunal un nombre un peu plus élevé de jeunes. Cependant, il se peut que le Canada ne reconnaisse pas autant de délinquants coupa-

6. Ces données ont été obtenues à la suite d'une demande spéciale à Statistique Canada. 


\section{FI G URE 9}

Taux de prise en charge formelle et informelle aux États-Unis

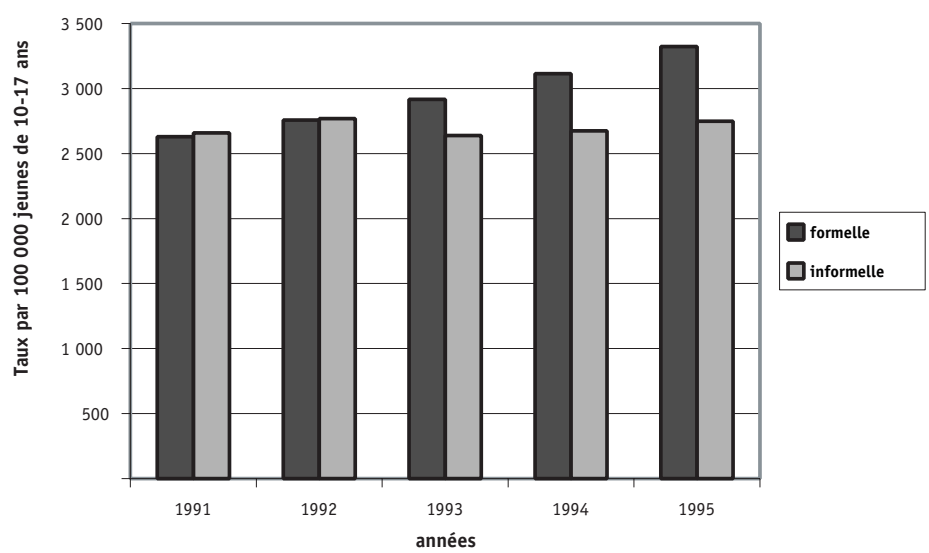

F I G URE 10

Taux d'arrestations des jeunes et taux des jeunes « non inculpés " (Canada)

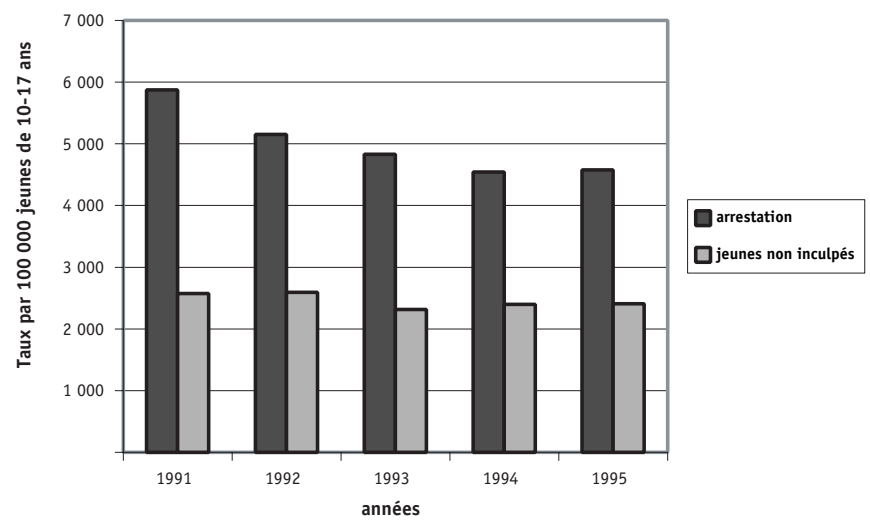


bles. Pour vérifier cette hypothèse, nous avons comparé le taux de cas de jeunes « reconnus délinquants » avec son équivalent canadien de « déclarations de culpabilité ».

Si l'on considère d'abord les infractions avec violence, on constate à nouveau que le Canada a un taux plus élevé (Figure 11). Cependant, toujours en ce qui concerne les infractions avec violence dont les auteurs ont été reconnus coupables, on s'aperçoit qu'au Canada le taux est resté relativement stable au cours des années, tandis qu'aux États-Unis, le taux a augmenté. Ainsi, en 1995, le taux de jeunes reconnus coupables d'infractions avec violence au Canada était plus élevé d'environ $6 \%$ que celui des États-Unis. Cependant, comme nous l'expliquerons plus loin, les États-Unis renvoient beaucoup plus de cas impliquant des jeunes aux tribunaux pour adultes. Il se peut donc que le nombre plus élevé de jeunes reconnus coupables par les tribunaux canadiens s'explique en partie par ce fait.

Pour les infractions contre la propriété, le Canada compte un taux plus élevé de jeunes reconnus coupables que les États-Unis (Figure 12). Alors que les États-Unis maintenaient un taux remarquablement stable au fil des années, le taux a diminué au Canada. En 1995, le Canada avait un taux de jeunes reconnus coupables de délits contre la propriété plus élevé d'environ $21 \%$ que celui des États-Unis.

Contrairement aux infractions avec violence et aux infractions contre la propriété, le Canada compte un taux plus bas de déclarations de culpabilité pour délits de drogue que les États-Unis (Figure 13). Au fil des années, on constate cependant que les taux canadiens augmentent de la même manière que ceux des États-Unis.

Mises sous garde. - Nous allons étudier maintenant les taux de mises sous garde pour chaque type d'infractions (violence, propriété et drogue). Pour calculer ces taux, le dénominateur choisi est le nombre total d'affaires (violence, propriété, drogue) de jeunes trouvés coupables (ou « reconnus délinquants» aux États-Unis) ${ }^{7}$. Pour le Canada, nous avons établi comme numérateur le nombre de placements en milieux

7. Ce dénominateur a été choisi parce qu'il évitait les problèmes liés au choix d'un dénominateur regroupant l'ensemble des jeunes dont l'âge va de 10 ans à l'âge maximum admissible au tribunal de la jeunesse. Comme nous l'avons déjà évoqué, une telle catégorie d'âge sous-évalue quelque peu l'activité des tribunaux de la jeunesse au Canada. En employant comme dénominateur le nombre de cas où il y a eu déclaration de culpabilité, nous pouvions, dans un certain sens, obtenir un portrait plus fidèle de ce qui se passait dans chaque pays. 


\section{F I G U RE 11}

Taux de déclaration de culpabilité pour tous les cas d'infractions avec violence

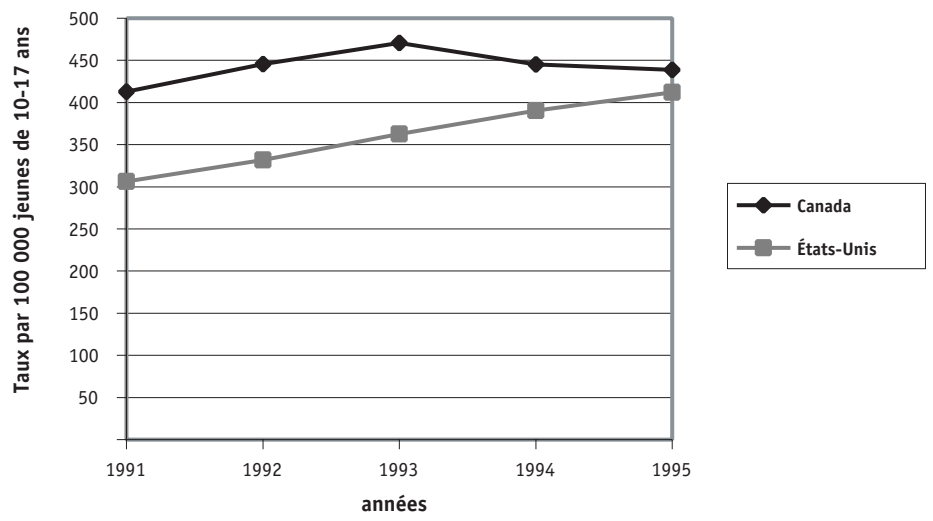

FIGURE 12

Taux de déclaration de culpabilité pour tous les cas d'infractions contre la propriété

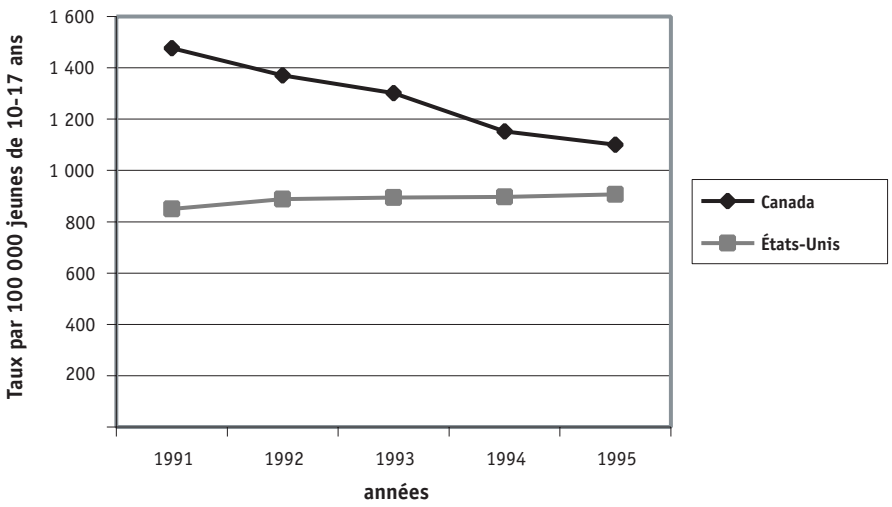


FIG URE 13

Taux de déclaration de culpabilité pour tous les cas d'infractions reliées à la drogue

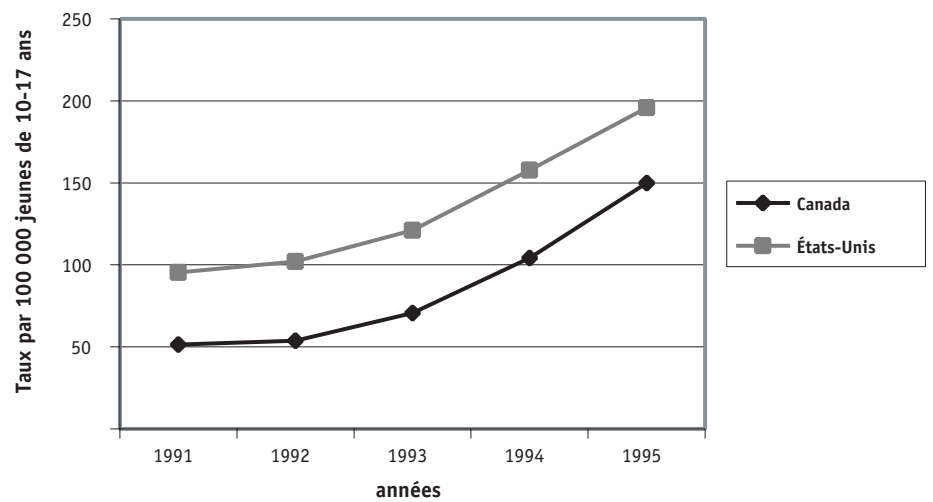

ouvert et fermé. Pour les États-Unis, le numérateur inclut l'ensemble des cas de jeunes reconnus délinquants et " placés », ce qui signifie que les jeunes ont été « placés» hors de leur foyer.

Nous avons constaté que le Canada avait recouru de plus en plus fréquemment à la mise sous garde dans les cas de violence alors qu'au contraire, les États-Unis avaient eu de moins en moins recours à la mise sous garde au fil des années (Figure 14). En 1995, le taux de mises sous garde pour infractions avec violence était presque le même dans les deux pays. Cependant, comme nous le mentionnerons plus loin, il existe un grand nombre de cas qui pourraient faire l'objet d'une sentence de mise sous garde mais qui sont renvoyés devant les tribunaux pour adultes aux États-Unis.

En ce qui concerne les infractions contre la propriété, le Canada a recours à la mise sous garde à un taux légèrement plus élevé que les États-Unis (Figure 15). Le taux canadien atteignit un sommet en 1994, puis il décrut quelque peu en 1995. En 1995, le Canada mettait les jeunes sous garde à un taux de 29 (pour 100 jeunes à l'égard desquels le tribunal rendait une décision sur la mesure à imposer) et les États-Unis mettaient sous garde à un taux de 25 pour cent.

Enfin, en matière d'infractions liées à la drogue, le Canada recourt à la mise sous garde à un taux plus bas que les États-Unis (Figure 16). En 


\section{F I G U RE 14}

Taux de mises sous garde des jeunes pour toutes les infractions avec violence

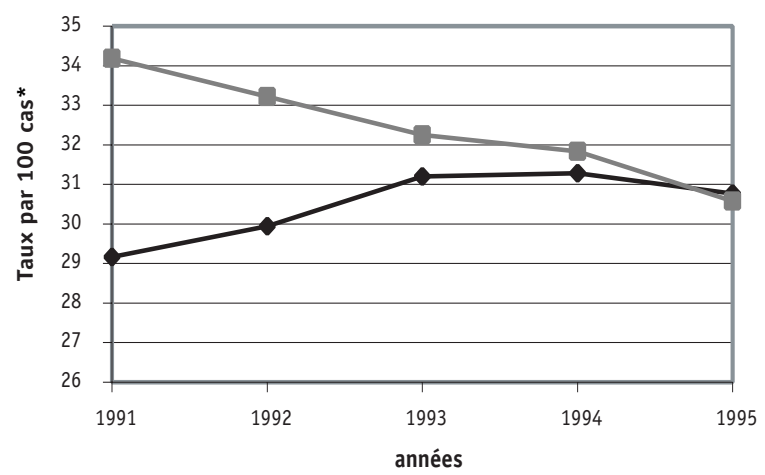

*cas où une décision a été prise sur une mesure (sentence)

FIGURE 15

Taux de mises sous garde pour toutes les infractions contre la propriété

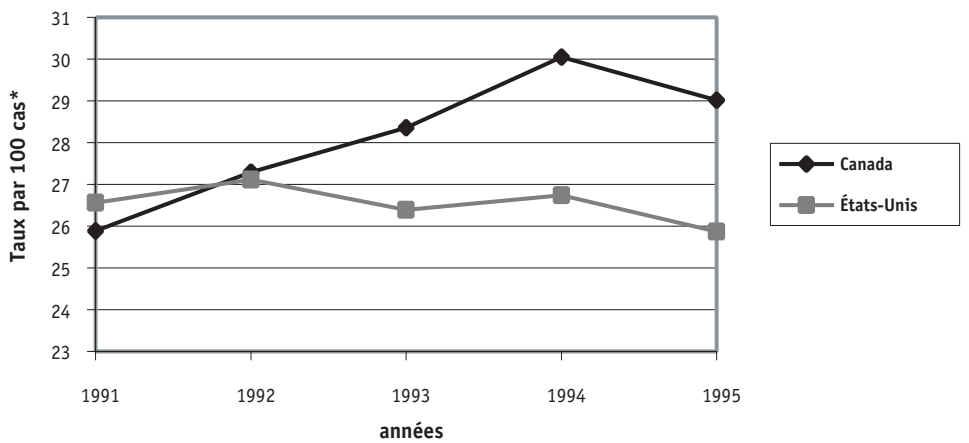

*cas où une décision a été prise sur une mesure (sentence) 
1995, le Canada avait un taux de mises sous garde d'environ $27 \%$ plus bas que celui des États-Unis. Contrairement aux infractions avec violence ou contre la propriété, les crimes reliés à la drogue étaient traités « de manière formelle » à un taux plus élevé aux États-Unis : de même, les jeunes étaient « reconnus délinquants » et mis sous garde dans de plus fortes proportions aux États-Unis. En ce qui concerne les infractions avec violence et contre la propriété, le Canada suivait la " filière formelle » et choisissait la mise sous garde à un taux similairement ou légèrement plus élevé que les États-Unis. Cette différence pourrait être liée à la «guerre contre la drogue » menée aux États-Unis : une telle " guerre » a en effet suscité une demande de traiter les problèmes de drogue en recourant plus souvent aux procédures officielles et en punissant «durement » les jeunes impliqués en les plaçant sous garde.

Une autre manière de comparer le recours à la mise sous garde dans chaque pays consiste à calculer le taux de mise sous garde pour 100000 jeunes de la population (âgés de 10 à 17 ans). En général, pour toutes les années dont nous avons tenu compte, le Canada a plus souvent utilisé la mise sous garde que les États-Unis (Tableau 2). Plus précisément, le Canada a des taux de mises sous garde constamment plus élevés que les États-Unis en matière d'infractions avec violence et d'infractions contre la propriété. Cependant, ce taux de mises sous garde pour 100000 jeunes semble être en voie de diminution au Canada pour les infractions avec violence et contre la propriété, alors qu'aux États-Unis, il semble être en augmentation, du moins pour ce qui concerne les infractions avec violence et les infractions reliées à la drogue.

Il ne faut pas vraiment s'étonner d'observer des tendances légèrement différentes lorsqu'on examine les taux de mises sous garde pour les cas trouvés coupables et les taux de mises sous garde pour 100000 jeunes présents dans la population. Le taux de mises sous garde pour 100000 jeunes de la population dépend de deux facteurs indépendants : les taux de déclaration de culpabilité pour une population de 100000 jeunes (Figures 11 à 13) et les taux de mises sous garde chez les jeunes trouvés coupables (Figures 14 à 16).

Renvois au tribunal pour adultes. - En ce qui concerne les décisions relatives au renvoi aux tribunaux pour adultes (pour tous les types de crime et pour 100000 jeunes âgés de 10 ans jusqu'à l'âge de la majorité pénale), nous constatons que les États-Unis transferent les cas aux tribunaux pour adultes 13 ou 14 fois plus souvent que le Canada (Tableau 3). 
FIGURE 16

Taux de mises sous garde pour toutes les infractions reliées à la drogue

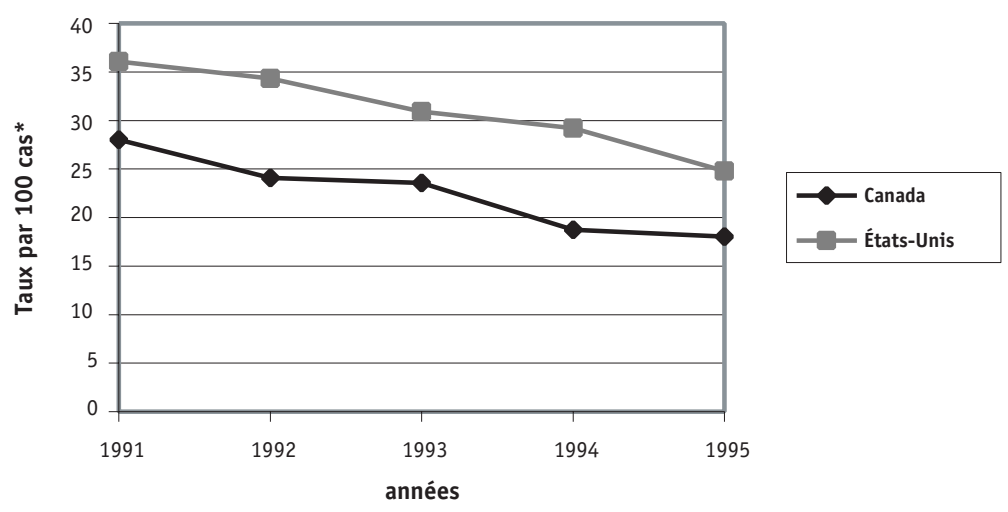

*cas où une décision a été prise sur une mesure (sentence)

TA BLEA U 2

Taux de mises sous garde au Canada et aux États-Unis

(par 100000 jeunes âgés de 10 à 17 ans)

\begin{tabular}{|l|l|c|c|c|c|c|}
\hline \multicolumn{2}{|c|}{} & 1991 & 1992 & 1993 & 1994 & 1995 \\
\hline \multirow{3}{*}{ Ensemble } & Canada & 763,11 & 779,35 & 820,58 & 799,37 & 764,24 \\
\cline { 2 - 7 } & É.-U. & 465,38 & 481,63 & 500,12 & 526,64 & 527,28 \\
\hline \multirow{2}{*}{ Violence } & Canada & 118,07 & 130,60 & 143,33 & 140,04 & 132,84 \\
\cline { 2 - 7 } & É.-U. & 104,75 & 110,17 & 116,96 & 124,28 & 126,07 \\
\hline \multirow{2}{*}{ Drogue } & Canada & 379,81 & 371,51 & 365,93 & 343,18 & 316,89 \\
\cline { 2 - 7 } & É.-U. & 237,55 & 240,82 & 236,13 & 239,91 & 234,43 \\
\cline { 2 - 7 } & Canada & 13,89 & 12,38 & 16,03 & 18,77 & 18,36 \\
\cline { 2 - 7 } & É.-U. & 34,42 & 34,99 & 37,40 & 46,11 & 48,51 \\
\hline
\end{tabular}


T A B LEA U 3

Taux* de cas transférés par décision judiciaire au Canada et aux États-Unis

\begin{tabular}{|lrrrrr|}
\hline Année & 1991 & 1992 & 1993 & 1994 & 1995 \\
\hline CANADA & 2,67 & 1,69 & 3,01 & 3,9 & 2,33 \\
ÉTATS-UNIS & 40,40 & 38,34 & 40,33 & 42,15 & 34,35 \\
\hline
\end{tabular}

* Les taux sont calculés pour 100000 jeunes âgés de 10 à 17 ans.

Il faut cependant se montrer prudent, car les chiffres relatifs aux États-Unis sous-évaluent substantiellement le taux réel de transferts. Il y a trois façons de transférer un cas au tribunal pour adultes aux ÉtatsUnis. La première est le « renvoi judiciaire », où le juge du tribunal des mineurs décide du renvoi. La seconde est le « renvoi statutaire », où le législateur établit dans la loi quelles infractions feront l'objet de renvois au tribunal pour adultes. Enfin, dans le cas du « renvoi par le procureur », il revient à ce dernier d'intenter la poursuite au tribunal pour adultes plutôt qu'au tribunal des mineurs. Or, on ne dispose pas de données nationales concernant les cas transférés par le biais des renvois statutaires et des renvois par le procureur.

On dispose d'indications à l'effet que la plupart des jeunes sont transférés par renvoi statutaire, ce qui inclut les jeunes âgés de moins de 18 ans envoyés à la cour criminelle parce qu'ils sont considérés comme des adultes dans leur État (Snyder et Sickmund, 1995). Par exemple, en 1995, 9700 jeunes furent transférés au tribunal pour adultes par renvoi judiciaire, alors que 176000 jeunes étaient transférés en raison d'un renvoi statutaire ${ }^{8}$ (Snyder et Sickmund, 1995 : 155). Les données que nous avons présentées concernent seulement les renvois judiciaires. Le Canada, au contraire, permet seulement le transfert d'un jeune à la suite d'une décision judiciaire. Le nombre de cas transférés au Canada entre 1991 et 1995 a varié d'un maximum de 123 à un minimum de 52. En additionnant les 176000 cas de renvois statutaires estimés aux 9700 cas de renvois judiciaires, les États-Unis obtiennent un taux de transferts de plus de 200 fois plus élevé que celui du Canada en 1995.

8. Il n'y pas d'estimations nationales disponibles pour le nombre de cas transférés à la suite d'un renvoi décidé par le procureur (Snyder et Sickmund, 1995). 


\section{Discussion}

D'après ce que nous venons de voir, des indices semblent appuyer l'affirmation de la ministre de la Justice, suivant laquelle le Canada recourrait à la mise sous garde à un taux plus élevé que les États-Unis. Cependant, une telle affirmation se doit d'être nuancée. Tout d'abord, les États-Unis renvoient des mineurs au tribunal pour adultes dans des proportions beaucoup plus élevées que le Canada. Ces cas ainsi transférés auraient pu subir une sentence de mise sous garde mais ne sont pas pris en compte lors du calcul des taux de mises sous garde. Les États-Unis ont donc moins recours à la mise sous garde pour mineurs que le Canada en partie parce que beaucoup de jeunes sont envoyés au tribunal pour adultes. Cette question des renvois doit être prise en compte lorsqu'il s'agit de comparer les taux de mises sous garde entre les deux pays.

De même, qu'il s'agisse de la mise sous garde ou, plus généralement, de l'utilisation du tribunal pour mineurs, un autre élément ajoute à la complexité de toute comparaison nationale : des variations considérables existent entre provinces et entre États, et ces variations sont largement obscurcies par les données. Comme nous l'avons souligné précédemment, le Canada est soumis à une seule loi fédérale, mais l'administration de cette loi varie énormément selon les provinces. Donc, si les estimations nationales des activités des tribunaux peuvent nous être utiles, elles ne peuvent pas être représentatives de l'activité d'une province. Pour saisir le fonctionnement détaillé du système de justice des mineurs, il faut rechercher comment chaque province a appliqué la loi.

Aux États-Unis, les estimations concernant l'activité des tribunaux de la jeunesse à l'échelon national masquent aussi les différences législatives et administratives entre États. Puisque chaque État définit en grande partie l'orientation de son propre système de justice des mineurs, on constate beaucoup plus de variations entre les États américains qu'entre les provinces canadiennes. Par exemple, les catégories d'âge admissibles au tribunal de la jeunesse varient suivant les États. Cette complexité doit être comprise et gardée à l'esprit lorsqu'on évoque ou lorsqu'on compare les données nationales. Ainsi, affirmer simplement que le Canada recourt plus souvent à la mise sous garde que les ÉtatsUnis peut se révéler trompeur - il faut tenir compte des complexités et des problèmes que suscitent de telles comparaisons.

Néanmoins, tout en tenant compte de telles complexités, ces données semblent offrir un appui à l'idée que le Canada recourt plus à la mise 
sous garde et, de manière générale, à son système de justice des mineurs que les États-Unis. Ainsi, on constate que les États-Unis ont des taux d'arrestations constamment plus élevés que le Canada - particulièrement lorsqu'il s'agit des formes les plus graves de violence. Cependant, en ce qui concerne les infraction avec violence et les infractions contre la propriété, on a constaté que le Canada soumettait les jeunes à une procédure formelle et les trouvait coupables à un taux plus élevé que les États-Unis. Une telle situation pourrait être due en partie au fait que les États-Unis traitent de manière informelle environ la moitié des cas signalés à la cour. Autre raison qui peut être invoquée : les États-Unis transferent de nombreux cas aux tribunaux pour adultes, ce qui fait que ces cas ne figurent pas dans les statistiques des tribunaux de la jeunesse. C'est pour les infractions reliées à la drogue que l'on constate dans les deux pays la plus forte augmentation d'arrestations et de déclarations de culpabilité. C'est seulement en matière de drogue que les taux de déclaration de culpabilité et de mises sous garde des États-Unis excèdent ceux $\mathrm{du}$ Canada. En matière d'infractions avec violence et d'infractions contre la propriété, le Canada recourt à la mise sous garde à un taux identique ou légèrement plus élevé que les États-Unis.

Nous ne disposons pas de comparaisons systématiques entre les États-Unis et le Canada pour ce qui concerne la durée de mise sous garde. Cependant, aux États-Unis en 1994, (l'année pour laquelle nous disposons des données les plus récentes), la durée moyenne de mise sous garde était d'environ cinq mois (Sickmund et al., 1997). Au Canada, en 1994-1995, la durée moyenne de séjour était d'environ 3 mois'.

Donc, contrairement à l'opinion répandue dans le public, les actes les plus graves de violence ne semblent pas avoir augmenté considérablement dans les deux pays de 1991 à 1996. De plus, on pourrait soutenir que la perception suivant laquelle la justice des mineurs est clémente au Canada ne repose sur aucun fondement empirique : il appert au contraire que le Canada recourt au système de justice des mineurs et à la mise sous garde à un taux semblable ou un peu plus élevé que les ÉtatsUnis, un pays reconnu pour ses hauts taux d'incarcération.

9. Cette donnée fut obtenue à la suite d'une demande spéciale à Statistique Canada. 


\section{Références}

Canadian Centre for Justice Statistics. 1992. Canadian Crime Statistics, 1991, Ottawa : Statistics Canada.

Canadian Centre for Justice Statistics. 1994. Canadian Crime Statistics, 1992, Ottawa : Statistics Canada.

Canadian Centre for Justice Statistics. 1994. Canadian Crime Statistics, 1993, Ottawa : Statistics Canada.

Canadian Centre for Justice Statistics. 1995. Canadian Crime Statistics, 1994, Ottawa : Statistics Canada.

Canadian Centre for Justice Statistics. 1996. Canadian Crime Statistics, 1995, Ottawa : Statistics Canada.

Canadian Centre for Justice Statistics. 1997. Canadian Crime Statistics, 1996, Ottawa : Statistics Canada.

Canadian Centre for Justice Statistics. 1992. Youth Court Statistics, 1991-1992, Ottawa : Statistics Canada.

Canadian Centre for Justice Statistics. 1994. Youth Court Statistics, 1992-1993, (Revised), Ottawa : Statistics Canada.

Canadian Centre for Justice Statistics. 1995. Youth Court Statistics, 1993-1994, Ottawa : Statistics Canada.

Canadian Centre for Justice Statistics. 1996. Youth Court Statistics, 1994-1995, Ottawa : Statistics Canada.

Canadian Centre for Justice Statistics. 1997. Youth Court Statistics, 1995-1996, Ottawa : Statistics Canada.

Doob, A. N. et SprotT, J. B. 1996. «Interprovincial variation in the use of youth courts », Canadian Journal of Criminology 38 (4) : 401-412.

Doob, A. N. et SprotT, J. B. 1998. "Is the "quality" of youth violence becoming more serious? »,Canadian Journal of Criminology 40 (2) : 185-194.

Doob, A. N. et SprotT, J. B. 1999. « Changes in youth sentencing in Canada », Federal Sentencing Reporter 11 (5) : 262-268.

Doob, A. N., Sprott, J. B., Marinos, V., et Varma, K. N. 1998. An exploration of Ontario residents' views of crime and the criminal justice system, Centre of Criminology : University of Toronto.

Mclellan, A. A. 1998. A Strategy for the Renewal of Youth Justice, Ottawa : Department of Justice.

Roberts, J. V. et Stalans, L. J. 1997. Public Opinion, Crime and Criminal Justice, Boulder, Colorado : Westview Press.

SicKMUnd, M., SNYder, H., et PIE-YAmagatA, E. 1997. Juvenile Offenders and Victims : 1997 Update on Violence, Washington, D.C. : Office of Juvenile Justice and Delinquency Prevention.

Sickmund, M. Stahl, A., Finnegan, T., Snyder, H., Poole, R. et Butts, J. 1998. Juvenile Court Statistics, 1995, Washington, DC : U.S. Department of Justice, Office of Juvenile Justice and Delinquency Prevention. 
SNYDER, H. 1997. Juvenile Arrests 1996, Washington, DC : U.S. Department of Justice, Office of Juvenile Justice and Delinquency Prevention.

SNYDER, H. et SicKMUND, M. 1995. Juvenile Offenders and Victims: A National Report, Washington, DC : U.S. Department of Justice, Office of Juvenile Justice and Delinquency Prevention.

Snyder, H., Finnegan, T., Stahl, A. et PoOle, R. 1997. Easy Access to Juvenile Court Statistics: 1986-1995 [Data presentation and analysis package], Washington, DC : Office of Juvenile Justice and Delinquency Prevention.

STAHL, A.L. 1998. Person Offences in Juvenile Court, 1986-1995, OJJDP Fact Sheet \#77. Washington DC : US Department of Justice, Office of Juvenile Justice and Delinquency Prevention.

TONRY, M. 1997. « Drug policies increasing racial disparities in U.S. prisons », p. 230-236 in Sentencing reform in overcrowded times: A comparative perspective, sous la direction de M. Tonry et K. Natlestad. Oxford : Oxford University Press. 